\title{
Management of computed tomography-detected pneumothorax in patients with blunt trauma: experience from a community-based hospital
}

Ashraf $F \underline{\text { Hefny }}^{1,2}$, MD, Fathima $T \underline{\text { Kunhivalappil }}{ }^{3}$, MD, Nikolay Matev ${ }^{3}$, MD, Norman A $\underline{\text { Avila }}^{4}$, BSN, Masoud O Bashir ${ }^{1}$, MD, Fikri M Abu-Zidan' ${ }^{2}$ MD, PhD

\begin{abstract}
INTRODUCTION Diagnoses of pneumothorax, especially occult pneumothorax, have increased as the use of computed tomography (CT) for imaging trauma patients becomes near-routine. However, the need for chest tube insertion remains controversial. We aimed to study the management of pneumothorax detected on CT among patients with blunt trauma, including the decision for tube thoracostomy, in a community-based hospital.

METHODS Chest CT scans of patients with blunt trauma treated at Al Rahba Hospital, Abu Dhabi, United Arab Emirates, from October 2010 to October 2014 were retrospectively studied. Variables studied included demography, mechanism of injury, endotracheal intubation, pneumothorax volume, chest tube insertion, Injury Severity Score, hospital length of stay and mortality. RESULTS CT was performed in 703 patients with blunt trauma. Overall, pneumothorax was detected on CT for 74 (10.5\%) patients. Among the 65 patients for whom pneumothorax was detected before chest tube insertion, 25 (38.5\%) needed chest tube insertion, while 40 (61.5\%) did not. Backward stepwise likelihood regression showed that independent factors that significantly predicted chest tube insertion were endotracheal intubation $(p=0.01)$, non-United Arab Emirates nationality $(p=0.01)$ and pneumothorax volume $(p=0.03)$. The receiver operating characteristic curve showed that the best pneumothorax volume that predicted chest tube insertion was $30 \mathrm{~mL}$.

CONCLUSION Chest tube was inserted in less than half of the patients with blunt trauma for whom pneumothorax was detected on CT. Pneumothorax volume should be considered in decision-making regarding chest tube insertion. Conservative treatment may be sufficient for pneumothorax of volume $<30 \mathrm{~mL}$.
\end{abstract}

Keywords: computed tomography, pneumothorax, trauma

\section{INTRODUCTION}

In an era of rapid advances in imaging technology and resource constraints, more studies are needed to define the role of chest computed tomography (CT) for patients with multiple blunt trauma. Advances in CT have greatly increased its diagnostic accuracy for acutely traumatised patients. ${ }^{(1)}$ Recent studies have demonstrated the value of chest CT for detecting chest injuries in spite of radiation exposure and cost. ${ }^{(2)}$ Thoracic injury is a major cause of trauma-related death. ${ }^{(3)}$ Routine use of chest CT for patients with multiple trauma is preferable to selective use, as it could change the course of patient management and improve outcomes. $^{(4)}$

Pneumothorax is the most common, potentially lifethreatening blunt chest injury. ${ }^{(5)}$ With the near-routine use of CT for imaging in patients with multiple trauma, the diagnosis of pneumothorax has increased. ${ }^{(1)}$ This is particularly so for occult pneumothorax, which is not detected on initial chest radiography. However, the need for chest tube insertion, which is an invasive procedure, for the management of small pneumothorax remains controversial, especially for patients with occult pneumothorax. ${ }^{(6)}$

According to Advanced Trauma Life Support (ATLS) guidelines, "Any traumatic pneumothorax is best treated with a chest tube. Neither general anaesthesia nor positive-pressure ventilation should be administered in the patient who has sustained a traumatic pneumothorax until a chest tube has been inserted ${ }^{\prime \prime} .^{(7)}$ In contrast, a prospective multicentre randomised controlled trial showed that in a haemodynamically stable patient, occult pneumothorax can be safely managed conservatively without chest tube insertion, even for mechanically ventilated patients. ${ }^{(1)}$ We aimed to study the management of CT-detected pneumothorax in patients with blunt trauma, including the decision to insert a chest tube, in a community-based hospital setting.

\section{METHODS}

All chest CT images of patients with blunt trauma who were treated at Al Rahba Hospital, Abu Dhabi, during a four-year period from October 2010 to October 2014 were retrospectively studied. Al Rahba Hospital is a community-based hospital, with a capacity of 190 beds, located on the main highway connecting Abu Dhabi and Dubai cities. About 700 patients with multiple trauma are admitted to the hospital annually.

Variables studied included demography, mechanism of injury, endotracheal intubation and mechanical ventilation, the need for chest tube insertion, Injury Severity Score (ISS), Glasgow Coma Scale (GCS) score, Revised Trauma Score, hospital length of stay

\footnotetext{
${ }^{1}$ Department of Surgery, Al Rahba Hospital, Abu Dhabi, ${ }^{2}$ Department of Surgery, College of Medicine and Health Sciences, UAE University, Al-Ain, ${ }^{3}$ Department of Radiology, ${ }^{4}$ Trauma Registry Program, Al Rahba Hospital, Abu Dhabi, United Arab Emirates

Correspondence: Dr Ashraf F Hefny, Assistant Professor, Trauma Group, Department of Surgery, College of Medicine and Health Sciences, UAE University, PO Box 18532 Main Building of Post Office, Al-Ain, United Arab Emirates. ahefny@uaeu.ac.ae
} 
and mortality. For patients who needed chest drainage, a chest tube of size 28-32 French was usually inserted using the open technique at the fifth intercostal space. Antibiotics were not routinely administered.

CT was performed using a General Electric 64-slice LightSpeed Volume (GE 64-slice LightSpeed VCT; General Electric, Boston, MA, USA). All patients underwent scanning of the entire thorax as part of trauma CT. Images were analysed in the lung, mediastinal and bone windows at $2.5-\mathrm{mm}$ thick axial cuts. CT images with pneumothorax were further analysed in the coronal and sagittal planes. The images were reviewed to determine the size of pneumothorax. The volume of pneumothorax was calculated in millilitres $(\mathrm{mL})$ using the manual segmentation method (software available in the CT machine). Radiological images (including chest radiographs and CT images) were independently reviewed by two radiologists. Patients with bilateral pneumothorax were analysed as one patient with two hemithoraces positive for pneumothorax. The volume of pneumothorax in patients having bilateral pneumothorax was calculated by adding up the pneumothorax volumes in the two hemithoraces.

The number of trauma patients who were treated in the emergency department and the number of trauma patients admitted were retrieved from the hospital's trauma registry. The Al Rahba Hospital Research Ethics Committee approved the research project (ARH/REC-040).

Non-parametric methods were used to compare the two independent groups due to the small number of patients recruited in the study. ${ }^{(8)}$ Fisher's exact test was used to compare categorical variables, while Mann-Whitney $U$ test was used to compare continuous or ordinal variables. Univariate analysis was used to compare patients with and without chest tube insertion subsequent to CT. Factors with $\mathrm{p}<0.1$ were then entered into a backward stepwise likelihood regression model. A p $\leq 0.05$ in the model was considered statistically significant. Data was analysed with IBM SPSS Statistics version 20 (IBM Corp, Armonk, NY, USA).

\section{RESULTS}

During the study period, 25,628 trauma patients were treated in the emergency department, among whom 2,860 (11.2\%) patients were admitted to the hospital.

Chest CT was performed for 703 patients with blunt trauma. Among these, pneumothorax was detected in 74 (10.5\%) patients and all of them were admitted to the hospital. Of these 74 patients, $15(20.3 \%)$ had bilateral pneumothorax and 63 (85.1\%) were men. The median age of the patients was 30 (range 11-74) years. Median ISS and GCS scores were 18 (range 8-45) and 15 (range 3-15), respectively. The most common mechanisms of injury were road traffic collision ( $n=63,85.1 \%)$ and fall from height $(n=7,9.5 \%)$.

Among the 74 patients, chest radiography was performed prior to CT for 40 (54.1\%) patients (Fig. 1). Overall, 9 (12.2\%) patients needed chest tube insertion before CT. For two patients with obvious tension pneumothorax on clinical examination, the chest tube was inserted prior to chest radiography. For another seven patients, the chest tube was inserted prior to CT but after chest radiography. Among these, six patients had pneumothorax

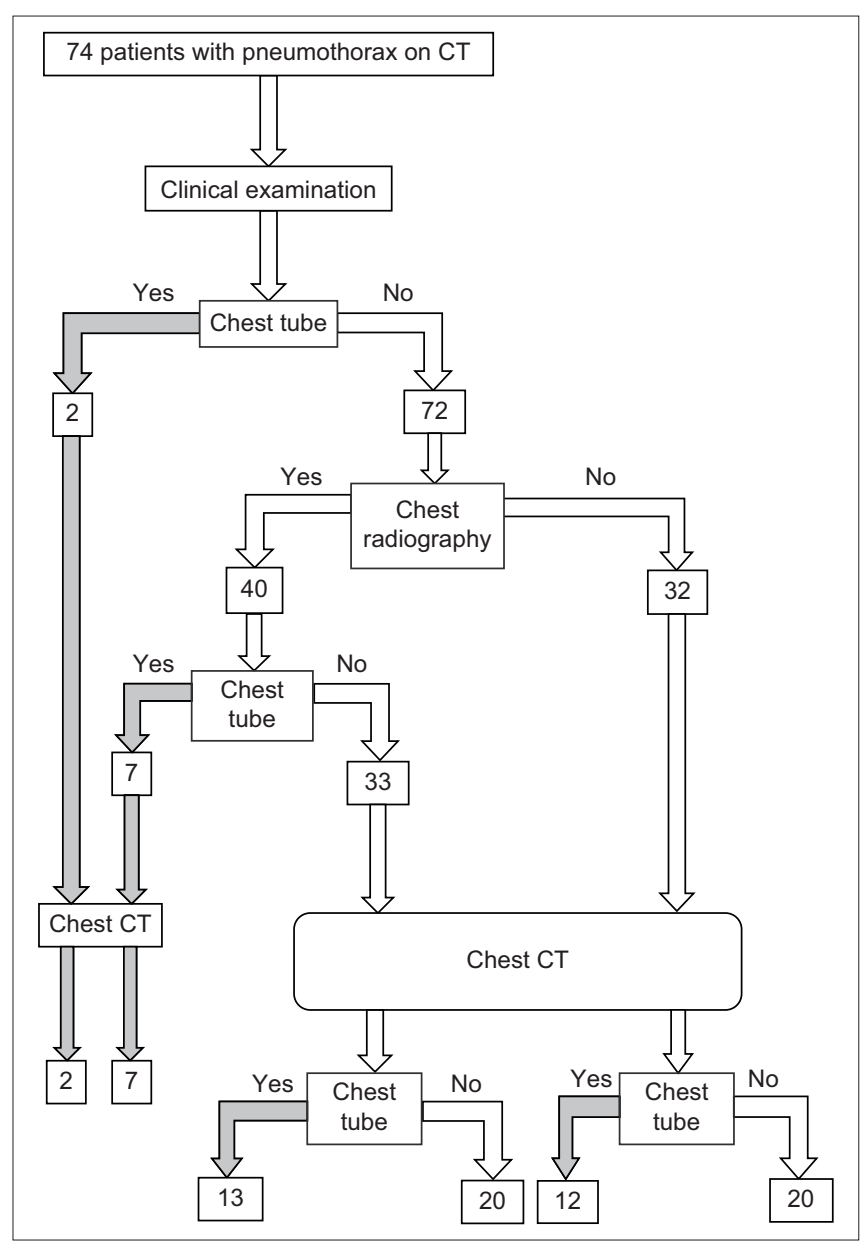

Fig. 1 Management algorithm of patients with blunt trauma $(n=74)$ for whom pneumothorax was detected on computed tomography (CT).

Table I. Radiological findings of patients with and without chest tube insertion after undergoing chest radiography following clinical examination.

\begin{tabular}{lccc}
\hline Finding & \multicolumn{2}{c}{ No. (\%) } & p-value* \\
\cline { 2 - 3 } & $\begin{array}{c}\text { Chest tube } \\
\text { insertion } \\
(\mathbf{n}=\mathbf{2 0})\end{array}$ & $\begin{array}{c}\text { No chest tube } \\
\text { insertion } \\
(\mathbf{n}=\mathbf{2 0})\end{array}$ & \\
\hline Normal & $2(10.0)$ & $7(35.0)$ & 0.13 \\
Pneumothorax & $9(45.0)$ & $1(5.0)$ & 0.008 \\
Haemothorax & $3(15.0)$ & $4(20.0)$ & 0.99 \\
Fractured ribs & $12(60.0)$ & $9(45.0)$ & 0.53 \\
Lung contusion & $7(35.0)$ & $6(30.0)$ & 0.99 \\
Subcutaneous & $5(25.0)$ & $2(10.0)$ & 0.41 \\
emphysema & & & \\
\hline
\end{tabular}

Patients could have more than one finding on chest radiography. ${ }^{*} \mathrm{p}$-values were calculated using Fisher's exact test.

on chest radiography and one patient, who was mechanically ventilated and had rib fractures on chest radiography, had the chest tube inserted prophylactically.

Table I compares the radiological findings of chest radiography that was performed following the initial clinical examination between patients with $(n=20)$ and without $(n=20)$ chest tube insertion. In the former group, chest tube insertion occurred before CT for seven patients and after CT for 13 patients. The only statistically significant difference between patients with and 
Table II. Univariate analysis of patients with and without chest tube insertion after pneumothorax was detected on CT.

\begin{tabular}{|c|c|c|c|}
\hline \multirow[t]{2}{*}{ Variable } & \multicolumn{2}{|c|}{ No. (\%) } & \multirow[t]{2}{*}{ p-value } \\
\hline & Chest tube insertion $(n=25)$ & No chest tube insertion $(n=40)$ & \\
\hline Age $(y r)^{*,+}$ & $29.0(18-58)$ & $33.5(11-74)$ & 0.58 \\
\hline Gender & & & 0.18 \\
\hline Men & $23(92.0)$ & $31(77.5)$ & \\
\hline Women & $2(8.0)$ & $9(22.5)$ & \\
\hline UAE & $5(20.0)$ & $18(45.0)$ & \\
\hline Non-UAE & $20(80.0)$ & $22(55.0)$ & \\
\hline Heart rate (beats/min) $)^{*,+}$ & $118.0(80-135)$ & $94.5(62-164)$ & 0.01 \\
\hline Mean systolic BP $(\mathrm{mmHg})^{*,+}$ & $127.5(66-190)$ & $128.5(47-187)$ & 0.95 \\
\hline GCS $^{*,+}$ & $15(3-15)$ & $15(3-15)$ & 0.02 \\
\hline ISS*,+ & $25(10-45)$ & $17(8-34)$ & 0.004 \\
\hline Respiratory rate*,+ & $21.5(0-48)$ & $22.0(11-40)$ & 0.52 \\
\hline RTS $^{*,+}$ & $12(8-12)$ & $12(6-12)$ & 0.11 \\
\hline Mechanism & & & 0.96 \\
\hline Road traffic collision & $23(92.0)$ & $34(85.0)$ & \\
\hline Fall & $1(4.0)$ & $4(10.0)$ & \\
\hline Other & $1(4.0)$ & $2(5.0)$ & \\
\hline Endotracheal intubation & $13(52.0)$ & $9(22.5)$ & 0.018 \\
\hline Fractured ribs & $19(76.0)$ & $27(67.5)$ & 0.58 \\
\hline ICU admission & $14(56.0)$ & $10(25.0)$ & 0.017 \\
\hline Hospital length of stay & $11(1-105)$ & $4(1-46)$ & 0.001 \\
\hline Pneumothorax volume $(\mathrm{mL})^{*, \neq}$ & $114.2(0.11-1,408.00)$ & $12.0(0.20-148.00)$ & 0.001 \\
\hline
\end{tabular}

Patients for whom chest tube was inserted prior to CT $(n=9)$ were excluded from the analysis. * Data presented as median (range). ${ }^{p} \mathrm{p}$-values were calculated using Fisher's exact test or Mann-Whitney $U$ test, as appropriate. $¥$ Volume of pneumothorax in both hemithoraces for each patient. BP: blood pressure; CT: computed tomography; GCS: Glasgow Coma Scale score; ICU: intensive care unit; ISS: Injury Severity Score; RTS: Revised Trauma Score; UAE: United Arab Emirates

Table III. Factors predicting chest tube insertion after CT based on backward likelihood logistic regression analysis.

\begin{tabular}{|c|c|c|c|c|c|}
\hline Variable & $\beta$ & Standard error & Wald test & p-value & OR $(95 \% \mathrm{CI})$ \\
\hline Non-UAE nationality & -3.700 & 1.43 & 6.72 & 0.01 & $0.03(0.001-0.410)$ \\
\hline Endotracheal intubation & 3.120 & 1.23 & 6.41 & 0.01 & $22.65(2.02-253.63)$ \\
\hline Pneumothorax volume & 0.017 & 0.01 & 4.66 & 0.03 & $1.02(1.00-1.03)$ \\
\hline Heart rate & 0.040 & 0.02 & 3.70 & 0.05 & $1.04(0.99-1.08)$ \\
\hline Constant & -5.190 & 2.03 & 6.53 & 0.01 & 0.006 \\
\hline
\end{tabular}

$\mathrm{Cl}$ : confidence interval; OR: odds ratio; UAE: United Arab Emirates

without chest tube insertion was the detection of pneumothorax on chest radiography ( $p=0.008$ using Fisher's exact test).

Chest radiography showed overt pneumothorax in only $10(25.0 \%)$ out of 40 patients: six had chest tube insertion before $\mathrm{CT}$, three had chest tube insertion after $\mathrm{CT}$ and one had no chest tube insertion. 30 (75.0\%) patients had occult pneumothorax (i.e. pneumothorax not detected by chest radiography), which was detected by CT. Among these patients, 11 (36.7\%) had chest tube insertion and 19 (63.3\%) did not. The median volume of occult pneumothorax on CT was 12.7 (range $0.11-1,401.00$ ) $\mathrm{mL}$. As chest tube insertion can change the exact volume of pneumothorax on subsequent $\mathrm{CT}$, patients with chest tube insertion were excluded from the logistic regression analysis.

Overall, pneumothorax was detected on CT in 65 patients without prior chest tube insertion. Among these, chest tube was inserted after CT for 25 (38.5\%) patients and not inserted for 40 $(61.5 \%)$ patients (Table II). Univariate comparison of these two groups showed that patients with chest tube had significantly higher heart rate $(p=0.01)$, lower GCS score $(p=0.02)$, higher injury severity $(p=0.004)$, more intensive care unit admission $(p=0.017)$ and higher need for endotracheal intubation $(p=0.018)$. Patients who needed chest tubes had significantly higher pneumothorax volume $(p=0.001)$ and hospital length of stay $(p=0.001)$. There was a trend towards chest tube insertion among non-UAE citizens, but this was not statistically significant $(p=0.06)$.

Factors with $p<0.1$ on univariate analysis were entered into a backward stepwise likelihood regression model (Table III). The model was highly significant ( $p<0.0001$ ), with a Nagelkerke $R^{2}$ of 0.6 . Independent factors that significantly predicted chest tube insertion among patients with blunt trauma were endotracheal intubation $(p=0.01)$, non-UAE nationality $(p=0.01)$ and pneumothorax volume $(p=0.03)$. The receiver operating characteristic (ROC) curve showed that the best pneumothorax 


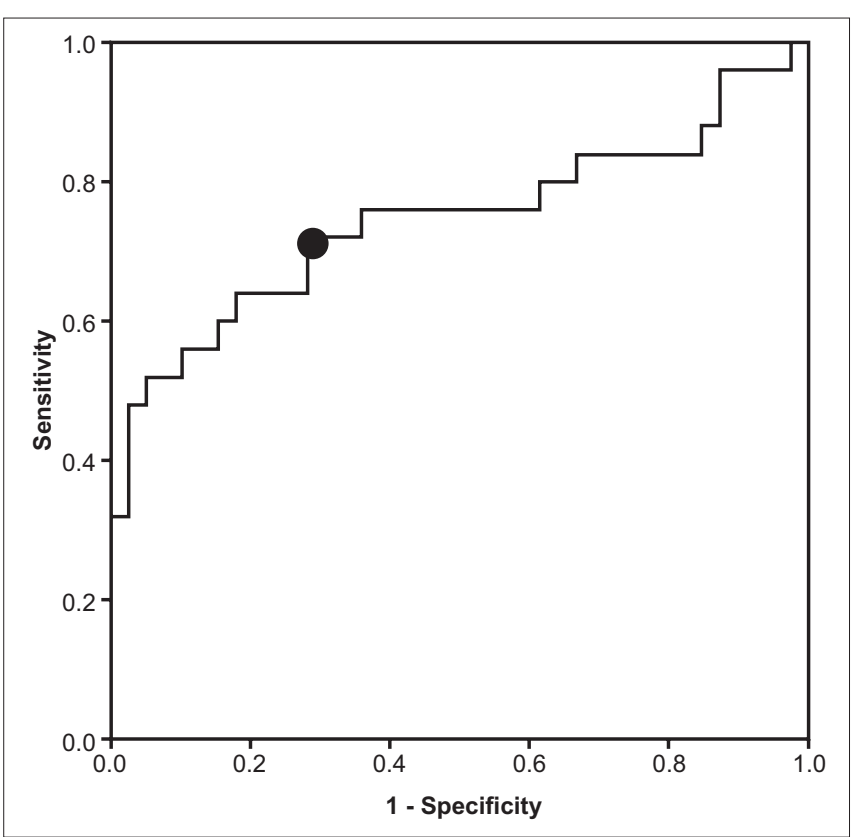

Fig. 2 Receiver operating characteristic curve for the best pneumothorax volume $(\mathrm{mL})$ that predicted chest tube insertion for patients in whom pneumothorax was detected on computed tomography.

volume that predicted chest tube insertion was $30 \mathrm{~mL}$ (sensitivity $72 \%$, specificity $72 \%$, area under the curve $75 \%$; Fig. 2 ).

Overall, 34 (45.9\%) patients had chest tube insertion and $40(54.1 \%)$ patients did not, instead receiving conservative treatment. There was no increase in the size of pneumothorax among patients who were treated conservatively following CT. These patients did not need any chest tube insertion for treatment of the pneumothorax. The median duration of chest tube insertion was 3 (range $1-7$ ) days. 30 (40.5\%) patients were admitted to the intensive care unit. Intubation and mechanical ventilation were needed for $28(37.8 \%)$ patients. The mean hospital length of stay was 9.8 (range 1-105) days. Five patients died, giving an overall mortality of $6.8 \%$.

\section{DISCUSSION}

Initial evaluation and treatment of our patients was based on ATLS principles. Supplemental imaging for stable patients with blunt trauma usually involves chest radiography as an adjunct to the primary survey and subsequent trauma CT as an adjunct to the secondary survey. ${ }^{(7)}$

While supine chest radiography cannot detect occult pneumothorax, supine oblique chest radiography may be as good as lung ultrasonography for the detection of large occult pneumothorax. ${ }^{(9)}$ However, if supine radiography of the chest shows abnormal findings, then the risk of chest injuries is considerably high. ${ }^{(10)}$ In a single-blind prospective clinical study, chest ultrasonography was more reliable for early bedside diagnosis of pneumothorax compared with supine chest radiography. ${ }^{(11)}$

Langdrof et al have shown, in a multicentre prospective study, that $71 \%$ of chest injuries would be missed if chest radiography was done without chest $\mathrm{CT}$ for occult injuries and that $14 \%$ of these occult injuries would need major interventions, including chest tube insertion for haemothorax or pneumothorax, mechanical ventilation or surgery. ${ }^{(2)} \mathrm{CT}$ is more sensitive and specific when compared to chest radiography and significantly reduces the time for patient management in emergency departments. ${ }^{(12)}$ In our study, chest radiography was not performed for $45.9 \%$ of patients, who were sent directly for trauma CT. This reflects the new trend over recent years of sending stable trauma patients for trauma CT immediately in order to save time and for more accurate diagnosis. ${ }^{(12,13)}$

The clinical importance and proper management of pneumothorax detected on CT in patients with blunt trauma remains controversial. ${ }^{(14,15)}$ In our study, pneumothorax was detected on CT among $10.5 \%$ of patients with blunt trauma, with $20.3 \%$ of these patients having bilateral pneumothorax. The most common mechanism of injury among our patients was road traffic collisions, similar to the findings of Mennicke et al. ${ }^{(5)}$ As in other studies, $75.0 \%$ of patients who underwent chest radiography in our study did not have clear evidence of pneumothorax on radiography although it was detected on subsequent CT. In these patients, pneumothorax might not have been diagnosed if CT had not been performed, persisting as occult pneumothorax instead. ${ }^{(16,17)}$ In all, $19(63.3 \%)$ out of 30 patients were treated conservatively without chest tube insertion in our study. Other studies have reported that $48 \%$ of patients with occult pneumothorax were treated conservatively. ${ }^{(3)}$ In comparison, we adopted a more conservative approach. None of our patients who were treated conservatively needed a chest tube insertion subsequently.

Similar to other studies, neither subcutaneous emphysema nor rib fractures on chest radiography were significantly associated with chest tube insertion in our patients. ${ }^{(18,19)}$ On the other hand, finding overt pneumothorax on chest radiography was significantly associated with chest tube insertion even prior to performing CT. This might be due to concerns among physicians of the possible development of tension pneumothorax during CT. In our study, CT was performed immediately after stabilisation of the patient and insertion of chest tube to detect other associated injuries. As the chest tube insertion may have resulted in mild pneumothorax that would not be picked up during subsequent $\mathrm{CT}$, we did not include these patients in the logistic regression analysis.

Our study showed that endotracheal intubation and pneumothorax volume were independent factors that significantly predicted chest tube insertion. ${ }^{(3,19)}$ ATLS training is mandatory for all trauma surgeons and emergency physicians in our hospital, who were likely to have followed the ATLS guidelines mandating chest tube insertion for intubated patients with traumatic pneumothorax. ${ }^{(7)}$

Chest drainage of mechanically ventilated patients with traumatic pneumothorax is controversial. Kirkpatrick et al have shown that the size of the pneumothorax is not important for chest tube insertion, while the need for prolonged ventilator support is. ${ }^{(1)}$ In contrast, other studies have shown that chest tube drainage is required when the size of the pneumothorax is $\geq 16 \mathrm{~mm}$ thick. The need for positive pressure ventilation alone does not justify chest drainage. ${ }^{(19)}$ Charbit et al have shown that $6 \%$ of patients with severe trauma had large occult pneumothorax (> $30 \mathrm{~mm}$ thick), which was not detected by chest radiography. The 
observed size and rate of accumulation of occult pneumothorax was not affected by the use of early mechanical ventilation. ${ }^{(20)}$ In a prospective observational study that included all blunt chest trauma patients requiring positive pressure ventilation, a majority of occult pneumothorax or haemothorax patients were carefully managed without tube thoracostomy. ${ }^{(21)}$ Studies have suggested that tube thoracostomy can be restricted to patients who had increased size of occult pneumothorax or haemothorax or to those who developed respiratory compromise. ${ }^{(21,22)}$

ROC curve is the recommended method for defining the best cut-off point with the highest sensitivity and specificity (usually at the point where the ROC curve becomes horizontal). Our cut-off point was at a pneumothorax volume of $30 \mathrm{~mL}$ (sensitivity $72 \%$, specificity $72 \%$ ). In our study, the decision for chest tube insertion was essentially based on clinical findings. None of our patients failed conservative management, indicating that we were very cautious in our decision-making. However, it is possible that the pneumothorax volume for which chest tube insertion is required could be over $30 \mathrm{~mL}$. Indeed, in a previous case report, we conservatively treated a clinically stable patient with a large volume of pneumothorax. ${ }^{(23)}$ We note that selective conservative management of chest trauma is the current trend. ${ }^{(24)}$ In addition, prophylactic antibiotics were not used in our study when inserting a chest tube, as its use is not justified for thoracostomy tube insertion. ${ }^{(25)}$

We were unable to explain why nationality was an independent factor that significantly affected chest tube insertion in our patients. We noticed that many stable UAE citizens with traumatic pneumothorax refused to have chest tube insertion, preferring to be treated conservatively. Similar to other studies, ISS, GCS score and heart rate were not independent predictors for chest tube insertion. (1) Hospital length of stay was significantly shorter among patients managed without chest tube insertion. ${ }^{(3)}$

One limitation of our study was that it was retrospective in nature and its sample size was relatively small. However, our experience from a community-based hospital is important, as over $50 \%$ of our patients with mild pneumothorax could be treated without chest tube insertion in this setting. This makes our findings generalisable to other similar tertiary hospitals. This notwithstanding, a future multicentre prospective study is needed for accurate detection of the size of the pneumothorax at which a chest tube can be safely avoided.

In conclusion, a chest tube was inserted in less than half of the patients with blunt trauma for whom pneumothorax was detected on CT. Pneumothorax volume should be included as a factor in the decision-making process prior to chest tube insertion for such patients. Among patients with blunt trauma, conservative treatment may be sufficient for pneumothorax of volume less than $30 \mathrm{~mL}$.

\section{ACKNOWLEDGEMENT}

We thank Mr Manoj Paul, Radiographer, Radiology Department, Al Rahba Hospital, Abu Dhabi, for his help with the measurements of pneumothorax volumes from the CT images.

\section{REFERENCES}

1. Kirkpatrick AW, Rizoli S, Ouellet JF, et al; Canadian Trauma Trials Collaborative and the Research Committee of the Trauma Association of Canada. Occult pneumothoraces in critical care: a prospective multicenter randomized controlled trial of pleural drainage for mechanically ventilated trauma patients with occult pneumothoraces. J Trauma Acute Care Surg 2013; 74:747-54

2. Langdorf MI, Medak AJ, Hendey GW, et al. Prevalence and clinical import of thoracic injury identified by chest computed tomography but not chest radiography in blunt trauma: multicenter prospective cohort study. Ann Emerg Med 2015; 66:589-600.

3. Wilson H, Ellsmere J, Tallon J, Kirkpatrick A. Occult pneumothorax in the blunt trauma patient: tube thoracostomy or observation? Injury 2009; 40:928-31.

4. Çorbacıoğlu SK, Erhan E, Aslan S, et al. The significance of routine thoracic computed tomography in patients with blunt chest trauma. Injury 2015; 46:849-53.

5. Mennicke M, Gulati K, Oliva I, et al. Anatomical distribution of traumatic pneumothoraces on chest computed tomography: implications for ultrasound screening in the ED. Am J Emerg Med 2012; 30:1025-31.

6. Yadav K, Jalili M, Zehtabchi S. Management of traumatic occult pneumothorax. Resuscitation 2010; 81:1063-8.

7. American College of Surgeons Committee. ATLS Advanced Trauma Life Support Program for Doctors American College of Surgeons. 9th ed. Chicago: American College of Surgeons, 2013.

8. Munro BH. Selected nonparametric techniques. In: Munro BH, ed. Statistical Methods for Health Care Research. 4th ed. New York: Lippincott Williams \& Wilkins, 2001: 97-121.

9. Matsumoto S, Sekine K, Funabiki T, et al. Diagnostic accuracy of oblique chest radiograph for occult pneumothorax: comparison with ultrasonography. World J Emerg Surg 2016; 11:5.

10. Raja AS, Mower WR, Nishijima DK, et al. Prevalence and diagnostic performance of isolated and combined NEXUS chest CT decision criteria. Acad Emerg Med 2016; 23:863-9.

11. Kaya Ş, Çevik AA, Acar N, et al. A study on the evaluation of pneumothorax by imaging methods in patients presenting to the emergency department for blunt thoracic trauma. Ulus Travma Acil Cerrahi Derg 2015; 21:366-72.

12. Healy DA, Hegarty A, Feeley I, et al. Systematic review and meta-analysis of routine total body CT compared with selective CT in trauma patients. Emerg Med J 2014; 31:101-8.

13. Hefny AF, Kunhivalappil FT, Matev N, et al. Usefulness of free intraperitoneal air detected by CT scan in diagnosing bowel perforation in blunt trauma: experience from a community-based hospital. Injury 2015; 46:100-4.

14. Johnson G. Traumatic pneumothorax: is a chest drain always necessary? J Accid Emerg Med 1996; 13:173-4.

15. Jenner R, Sen A. Best evidence topic report. Chest drains in traumatic occult pneumothorax. Emerg Med J 2006; 23:138-9.

16. Cai W, Lee JG, Fikry K, et al. MDCT quantification is the dominant parameter in decision-making regarding chest tube drainage for stable patients with traumatic pneumothorax. Comput Med Imaging Graph 2012; 36:375-86.

17. Lee RK, Graham CA, Yeung JH, Ahuja AT, Rainer TH. Occult pneumothoraces in Chinese patients with significant blunt chest trauma: radiological classification and proposed clinical significance. Injury 2012; 43:2105-8.

18. Ball CG, Ranson K, Dente CJ, et al. Clinical predictors of occult pneumothoraces in severely injured blunt polytrauma patients: a prospective observational study. Injury 2009; 40:44-7.

19. Notrica DM, Garcia-Filion P, Moore FO, et al. Management of pediatric occult pneumothorax in blunt trauma: a subgroup analysis of the American Association for the Surgery of Trauma multicenter prospective observational study. J Pediatr Surg 2012; 47:467-72.

20. Charbit J, Millet I, Maury C, et al. Prevalence of large and occult pneumothoraces in patients with severe blunt trauma upon hospital admission: experience of 526 cases in a French level 1 trauma center. Am J Emerg Med 2015; 33:796-801.

21. Mahmood I, Tawfeek Z, El-Menyar A, et al. Outcome of concurrent occult hemothorax and pneumothorax in trauma patients who required assisted ventilation. Emerg Med Int 2015; 2015:859130.

22. Zhang M, Teo LT, Goh MH, Leow J, Go KT. Occult pneumothorax in blunt trauma: is there a need for tube thoracostomy? Eur J Trauma Emerg Surg 2016; 42:785-90.

23. Idris BM, Hefny AF. Large pneumothorax in blunt chest trauma: is a chest drain always necessary in stable patients? A case report. Int J Surg Case Rep 2016; 24:88-90.

24. Kong VY, Oosthuizen GV, Clarke DL. Selective conservatism in the management of thoracic trauma remains appropriate in the 21 st century. Ann R Coll Surg Engl 2015; 97:224-8.

25. Kong VY, Sartorius B, Oosthuizen GV, Clarke DL. Prophylactic antibiotics for tube thoracostomy may not be appropriate in the developing world setting. Injury 2015; 46:814-6. 